\title{
Fracture Mechanics Analyses of Reinforced Carbon-Carbon Wing-Leading-Edge Panels
}

\author{
Ivatury S. Raju ${ }^{1}$ \\ NASA Langley Research Center, Hampton, Virginia \\ Dawn R. Phillips ${ }^{2}$ \\ NASA Marshall Space Flight Center, Huntsville, Alabama \\ Norman F. Knight, Jr. ${ }^{3}$ \\ General Dynamics - Advanced Information Systems, Chantilly, Virginia \\ and \\ Kyongchan Song ${ }^{4}$ \\ ATK Space Division, Hampton, Virginia
}

\begin{abstract}
Fracture mechanics analyses of subsurface defects within the joggle regions of the Space Shuttle wing-leading-edge RCC panels are performed. A 2D plane strain idealized joggle finite element model is developed to study the fracture behavior of the panels for three distinct loading conditions - lift-off and ascent, on-orbit, and entry. For lift-off and ascent, an estimated bounding aerodynamic pressure load is used for the analyses, while for onorbit and entry, thermo-mechanical analyses are performed using the extreme cold and hot temperatures experienced by the panels. In addition, a best estimate for the material stressfree temperature is used in the thermo-mechanical analyses. In the finite element models, the substrate and coating are modeled separately as two distinct materials. Subsurface defects are introduced at the coating-substrate interface and within the substrate. The objective of the fracture mechanics analyses is to evaluate the defect driving forces, which are characterized by the strain energy release rates, and determine if defects can become unstable for each of the loading conditions.
\end{abstract}

\section{Introduction}

$\mathrm{R}$ EINFORCED carbon-carbon (RCC) is used in the wing-leading-edge (WLE) panels of the Space Shuttle orbiter wings. The RCC in the panels provides thermal protection from the intense heat during entry of the Orbiter Vehicle. RCC is a composite laminated structure and consists of substrate material between two layers of coating. During manufacturing, craze cracks form in the coating. Each of the RCC panels has two joggle regions, which aid in assembly of the WLE. The regions between the joggles of the panels are called acreage regions.

During the lift-off and ascent phase of flight, the panels experience mechanical loads and negligible thermal loading. During the on-orbit phase of flight, the panels experience a severe cold soak, with negligible mechanical loads, and during entry, the panels experience extreme heating, with negligible mechanical loads. As such, the liftoff and ascent phases are predominantly mechanical loading cases, while the on-orbit and entry phases are predominantly thermal loading cases. Thus, mechanical loading and thermal loading cases can be analyzed separately.

In this paper, a two-dimensional (2D) plane strain idealized joggle finite element model is developed to study the fracture behavior of the panels under three phases of flight - lift-off and ascent, on-orbit, and entry. ${ }^{1-2}$ Studies

\footnotetext{
${ }^{1}$ NASA Technical Fellow for Structures, NASA Engineering and Safety Center. Fellow AIAA, Member ASME.

${ }^{2}$ Aerospace Engineer. Senior Member AIAA.

${ }^{3}$ Chief Engineer, Structural Mechanics. Fellow AIAA, Fellow ASME.

${ }^{4}$ Structural Analyst. Member AIAA.
} 
involving the lift-off and ascent phases of flight with mechanical loads and studies involving the entry and on-orbit phases of flight with thermal loading conditions are presented. Fracture mechanics analyses are performed assuming that subsurface defects are present in the joggle region either at the coating-substrate interface or within the substrate. The objective of the fracture mechanics analyses is to evaluate the defect driving forces, which are characterized by the strain energy release rates, and determine if subsurface defects can become unstable.

\section{Analysis}

Global three-dimensional (3D) models of a WLE panel that had higher fidelity along the joggle regions than elsewhere in the panel were developed in Reference 3. The higher fidelity along the joggle region included discrete layer modeling of the coating and substrate material through the RCC thickness. The global 3D model was then analyzed for entry heating cases with a temperature profile. No "hot spots" were identified on the panel. However, increased stress levels were observed all along one of the joggles; i.e., this increase in stress was independent of the location around the WLE. The analysis also showed that this increase in stress in the joggle was dependent on the local temperature. Results from a global 3D analysis for a specified uniform temperature confirmed that the increase in stress in the joggle region was not dependent on the location around the WLE.

Results from the global 3D panel model were compared with results from an independent local 3D model. ${ }^{4}$ The through-the-thickness stresses and strains were determined to be comparable for the two models using the simple boundary conditions for the local 3D model. These local 3D models also showed that the stresses do not exhibit any variation around the WLE except near the specimen edges. Thus, it was concluded that a two-dimensional slice through the RCC thickness near the joggle could be modeled using two-dimensional (2D) plane strain assumptions to study the behavior of the joggle for the loading conditions for each of the three phases of flight. In this paper, such a 2D plane strain joggle model is pursued.

\section{A. Finite Element Model}

The details of the 2D plane strain idealized joggle finite element (FE) model are shown in Figure 1. The model is 4 in. wide and has a constant thickness of 0.24 in. The substrate is 0.16 in. thick, and the coating layers have a total thickness of 0.08 in. The ABAQUS ${ }^{5}$ CPE4I elements are used in the analyses. ${ }^{5}$ There are ten elements through the thickness of the substrate ( $z$ direction) and four elements through the thickness of each of the coating layers. In the $x$ direction, the element spacing is set at $\sim 1 / 32$ in. ( $\sim 0.031$ in.) in the flat regions away from the joggle and $\sim 1 / 64$ in. ( $\sim 0.016$ in.) in the joggle region. The basic model (before any modifications for subsurface defects, etc. are made) has 3743 nodes and 3528 elements. The node at the bottom left of the model (on the inner mold line or IML) is constrained in both the $x$ and $z$ directions $\left(u_{x}=u_{z}=0\right)$, and the node on the bottom right is constrained in the $z$ direction.

The substrate and coating are modeled as two separate materials in the FE model. The coating is modeled with craze cracks explicitly included in the mesh, creating "islands" of coating material (see Figure 1). These craze cracks are assumed to be oriented vertically (i.e., parallel to the $z$ axis). For the fracture mechanics analyses, a subsurface defect is inserted either along the coating-substrate interface or just within the substrate material, as shown in Figure 2.

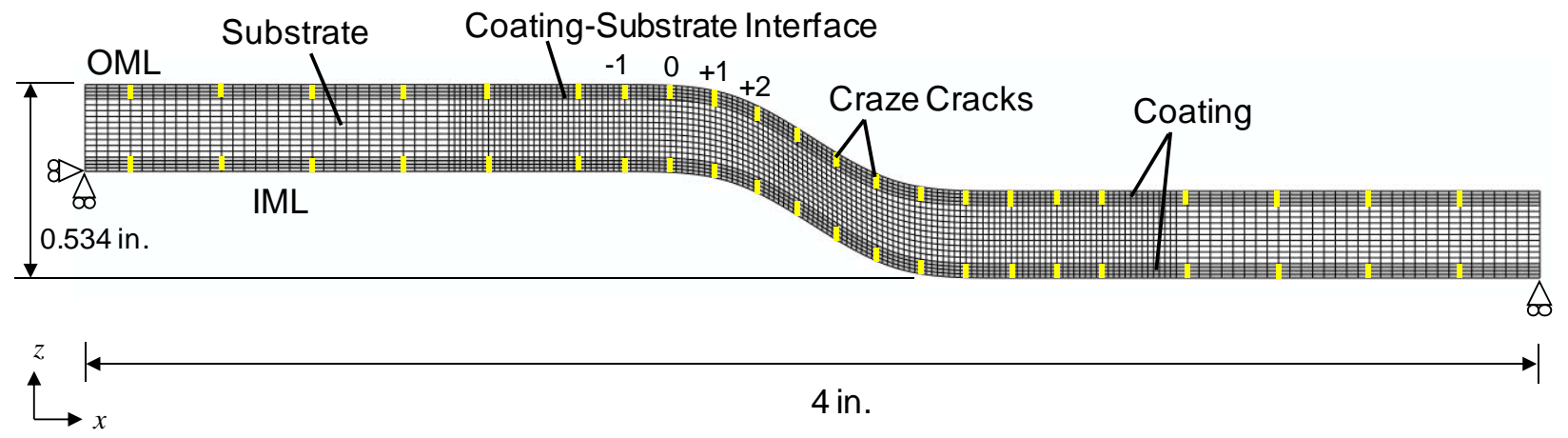

Figure 1. Idealized joggle finite element model.

\footnotetext{
${ }^{5}$ ABAQUS is a registered trademark of Dassault Systèmes.
} 


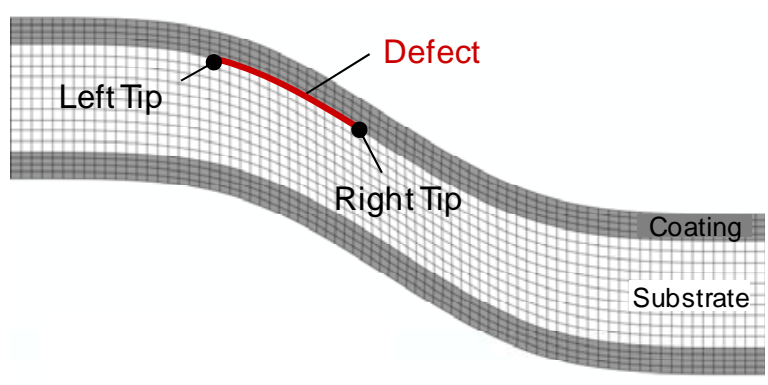

(a) Interface defect

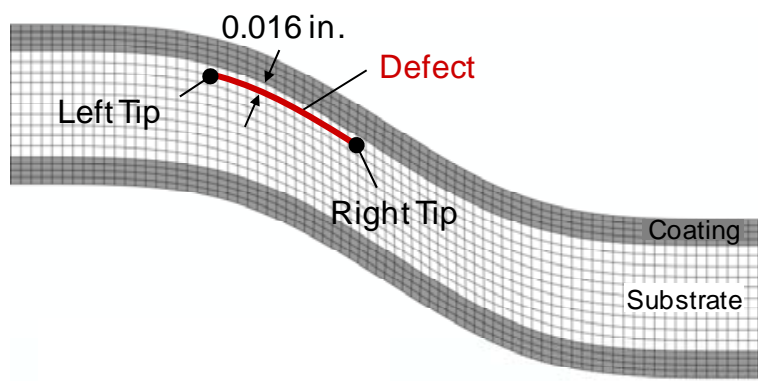

(b) Substrate defect

Figure 2. Defects inserted at the coating-substrate interface and 0.016 in. into the substrate in the joggle model.

The method of defect insertion is demonstrated in Figure 3. To insert a subsurface defect between elements A-D and E-H, nodes 2-4 are duplicated to create new nodes $2^{\prime}-4$ '. The connectivity of elements A-D is left unaltered, while the connectivity of elements E-H is modified to use the new nodes $2^{\prime}-4$ '. These new nodes are initially coincident with the original nodes; i.e., both sets of nodes have identical coordinates. Nodes 1 and 5 define the left and right defect tips, respectively. As the finite element solution progresses, the initially coincident free surfaces that define the defect are allowed to move relative to each other. Depending on the loading, the surfaces may separate, as shown in Figure 3, or come into contact. In the cases where contact may occur, contact conditions are defined on the defect surfaces to prevent interpenetration. The craze cracks are inserted in the same way as the subsurface defects; duplicate nodes are introduced, and contact is included between the elements that define the craze crack surfaces. For all of the analyses in this report, the value of the coefficient of friction $\mu$ for the contact condition used for the craze crack surfaces is $\mu=0.05$, and $\mu=0$ for the defect surfaces..

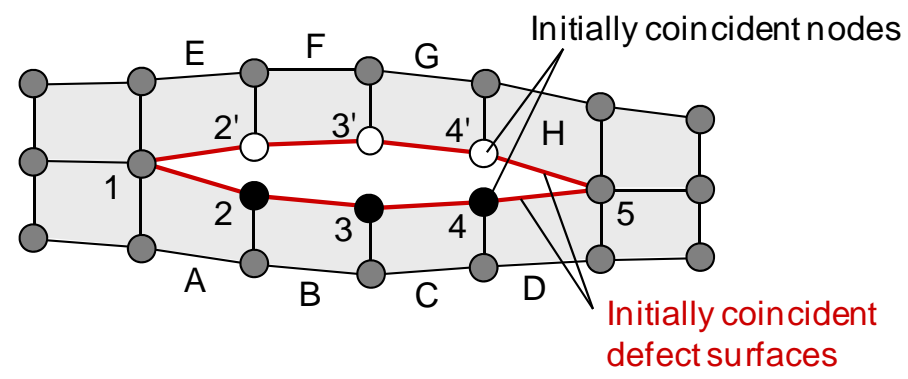

Figure 3. Defect insertion in the finite element models.

The craze cracks in the coating at the outer mold line or OML are numbered consecutively from Location 0 , as shown in Figure 1. Craze crack Location 0 is defined as the location where the transition from the flat acreage region to the curved joggle begins. Then, the craze cracks in the joggle region (to the right of Location 0 ) are numbered with positive numbers, while the craze cracks in the acreage (to the left of Location 0 ) are numbered with negative numbers. This notation is adopted for convenience.

\section{B. Defect Driving Force}

In all the fracture mechanics analyses presented in this paper, the defect driving force is characterized by the strain energy release rates. The total strain energy release rate $G_{\mathrm{T}}$ is the energy required to create a new unit area of the defect surface. The strain energy release rates are evaluated using the virtual crack closure technique (VCCT). In finite element analysis, the VCCT uses the nodal forces at the node at the defect tip and the displacements at the nodes behind the defect tip. ${ }^{6-8}$ In the analyses in this paper, the FE models were developed such that the element edge length $(\Delta a)$ of the elements ahead and behind the defect tip was of equal size. Figure 4 shows local modeling at the defect tip in a $2 \mathrm{D}$ analysis. The defect tip is represented by node $i$. Elements $I$ and $J$ contribute to the internal forces, $Z_{i}$ and $X_{i}$, at node $i$. The internal forces at node $i$ and the relative displacements between nodes $j$ and $k, \Delta w_{j, k}=w_{j}-w_{k}$ and $\Delta u_{j, k}=u_{j}-u_{k}$, are used to evaluate the individual $G$ values as

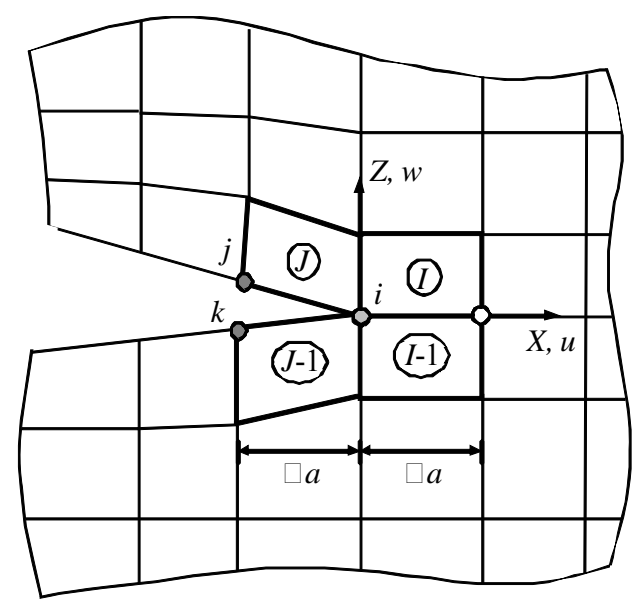

Figure 4. VCCT scheme for 4-node (linear) 2D elements. 


$$
G_{\mathrm{I}}=\frac{Z_{i} \Delta w_{j, k}}{2 \Delta a}, \quad G_{\mathrm{II}}=\frac{X_{i} \Delta u_{j, k}}{2 \Delta a}
$$

In Equation $1, G_{I}$ and $G_{\text {II }}$ are the Mode I and Mode II strain energy release rates, the $G$ values. The total strain energy release rate $G_{\mathrm{T}}$ can be evaluated using

$$
G_{\mathrm{T}}=G_{\mathrm{I}}+G_{\mathrm{II}}
$$

The $G$ values determined from Equations (1) and (2) are compared to the Mode I and II fracture toughness values, $G_{I c}$ and $G_{I I c}$. In this paper, a conservative comparison is made by comparing the total strain energy release rate $G_{\mathrm{T}}$ to the Mode I fracture toughness $G_{\mathrm{Ic}}$, which is the smallest of the fracture toughness values for the different modes. Unstable defect growth may occur when $G_{\mathrm{T}}$ is greater than or equal to $G_{\mathrm{I}}$; i.e., $G_{\mathrm{T}} / G_{\mathrm{Ic}} \geq 1.0$. However, when this ratio exceeds unity, further examination of the individual $G$ values and the fracture mode mixity is required to determine whether the defect configuration is stable or not for the given loading.

\section{Lift-Off and Ascent Loading}

During lift-off and ascent, a coating segment above an existing subsurface defect experiences acoustic and vibratory pressure loads and loads due to maximum dynamic pressure. An estimate of the bounding loads for lift-off and ascent was performed, and the details are presented in Reference 1. A pressure load equal to the estimated bounding load, $p_{\mathrm{L \& A}}$, is assumed to act normal to the coating segment over an assumed defect, as illustrated in Figure 5, and is used for all of the lift-off and ascent analyses in this paper. For convenience in presentation, loading to this bounding pressure load will be referred to as "lift-off".

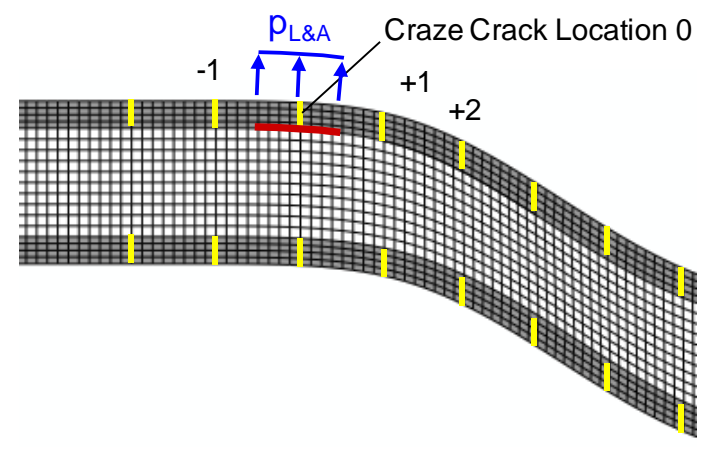

Figure 5. Pressure load for lift-off and ascent analyses.

\section{Entry and On-Orbit Loading}

During entry, the Space Shuttle is subjected to very high temperatures, with a peak-heating entry temperature occurring after the entry interface. In this paper, the maximum peak-heating temperature, $T_{\text {entry }}$, is assigned as the final temperature for the analyses as it poses the worst entry thermal condition. For convenience in presentation, peak heating to this maximum entry temperature will be referred to as "entry". For the entry analyses in this paper, at-temperature material properties at $T_{\text {entry }}$ are used for the coating and the substrate.

While the Space Shuttle is in orbit, it experiences a heating/cooling thermal cycle. In this paper, the coldest temperature of this thermal cycle, $T_{\text {on-orbit }}$, is assigned as the final temperature for the analyses as it poses the worst on-orbit thermal condition. For convenience in presentation, cooling to this on-orbit extreme cold temperature will be referred to as "on-orbit". For the on-orbit analyses in this paper, at-temperature material properties at $T_{\text {on-orbit }}$ are used for the coating and the substrate.

\section{E. Stress-Free Temperature}

Thermo-mechanical analysis results of RCC structures are significantly influenced by the stress-free temperature (SFT) of the material. ${ }^{9}$ An analysis process to determine a single effective best-estimate value of SFT for both entry and on-orbit was described in Reference 10. This effective best-estimate SFT value is used in the thermomechanical analyses of the entry and on-orbit loading conditions in this paper. The SFT plays an important role in the thermo-mechanical analyses as it directly influences the thermal loading $\Delta T, \Delta T=T$-SFT, applied to the model. In situations where the structure is subjected to cold temperatures, a high SFT yields a larger value of $\Delta T$, while in situations where the structure is subjected to elevated temperatures, a low SFT yields a larger value of $\Delta T$.

\section{Results and Discussion}

In this section, the defect driving forces, $G$ values, are presented first for interface defects and then for substrate defects. Lift-off and ascent, entry, and on-orbit loading conditions are evaluated for both interface and substrate defects. On-orbit and entry thermal loadings are evaluated using the best-estimate SFT value. The total defect 
driving force is compared to the Mode I fracture toughness to identify unstable subsurface defect configurations and loading conditions.

\section{A. Interface Defects}

\section{Baseline-I Configuration}

The baseline configuration for interface defects is denoted as Baseline-I for convenience in presentation. The Baseline-I model is shown in Figure 6. This model has a 0.125-in. interface defect centrally located under the craze crack at Location 0, as shown in Figure 6(a); the left tip is midway between craze crack Locations -1 and 0 (i.e., at Location $-1 / 2$; the acreage side), and the right tip is midway between Locations 0 and +1 (i.e., at Location $+1 / 2$; the joggle side). Because this defect is located at the coating-substrate interface, the craze crack at this location has two completely free surfaces that do not connect at the coating-substrate interface, as shown in Figure 6(b).

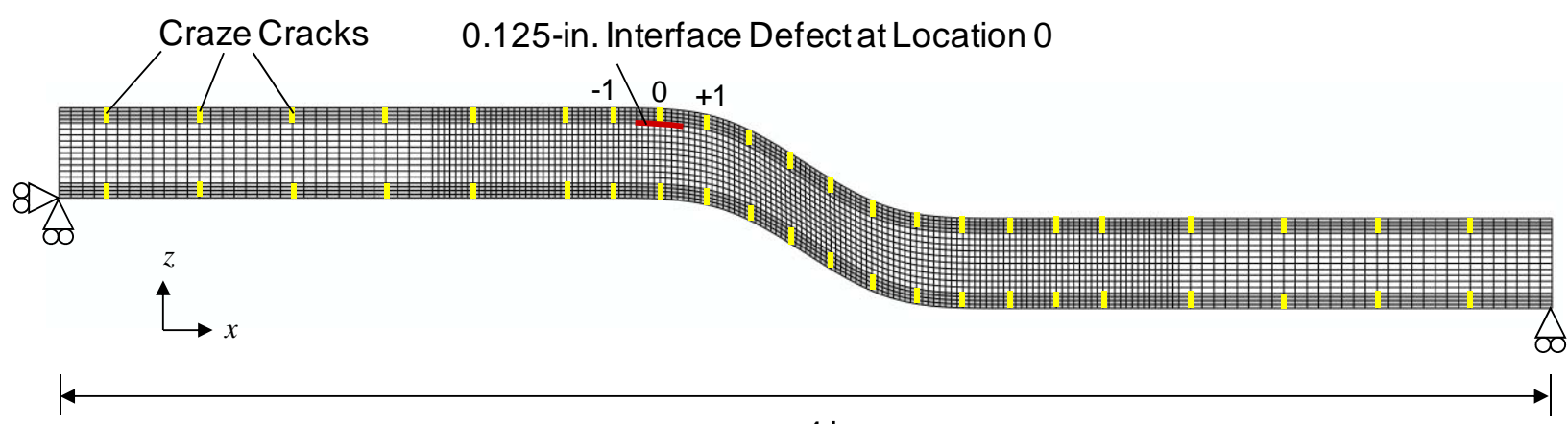

4 in.

(a) Finite element model

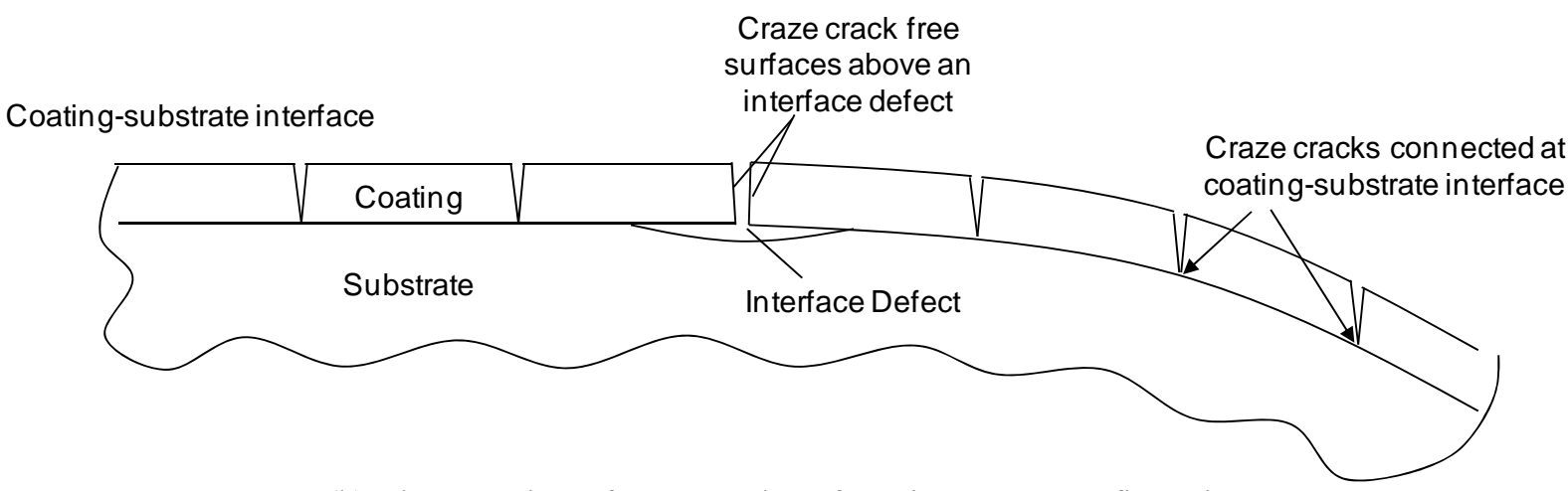

(b) Close-up view of craze crack surfaces in an open configuration

Figure 6. Baseline-I model.

For lift-off and ascent, the deformed configuration (at 500 times magnification) is shown in Figure 7(a). The pressure load pulls the coating material upward (in the positive $z$ direction), opening the defect. Because the pressure load is applied normal to the coating surface, a small amount of bending is also observed. Because of this bending, the two craze crack surfaces could come into contact. (Note that Figure 7(a) does not show contact.) The left and right tip $G_{\mathrm{T}} / G_{\mathrm{Ic}}$ values for the Baseline-I model subjected to the lift-off loading condition are essentially zero (less than 0.001), indicating that the defect is stable and unlikely to grow.

For entry, the deformed configuration (at twenty times magnification) is shown in Figure 7(b). Due to the peakheating temperature, as the coating material expands, the two craze crack surfaces above the defect come into contact. After contact, as the coating continues to expand, the craze crack surfaces press together and deform relative to each other. The coating material to the right of the craze crack displaces upward (in the positive $z$ direction) as it is pushed away by the coating material to the left of the craze crack trying to expand further in the positive $x$ direction. The left and right tip $G_{\mathrm{T}} / G_{\mathrm{Ic}}$ values for the Baseline-I model subjected to the entry loading condition are 0.005 and 0.31 , respectively, indicating that the defect is stable and unlikely to grow. 


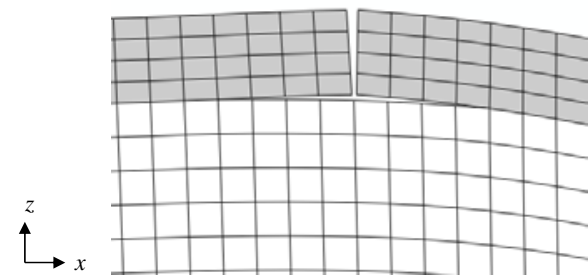

(a) Lift-Off (500x)

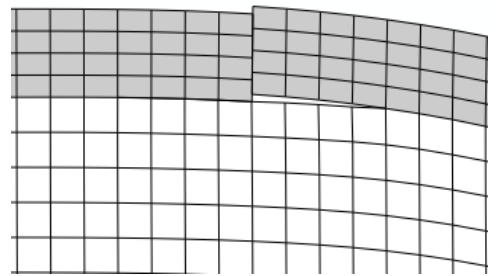

(b) Entry (20x)

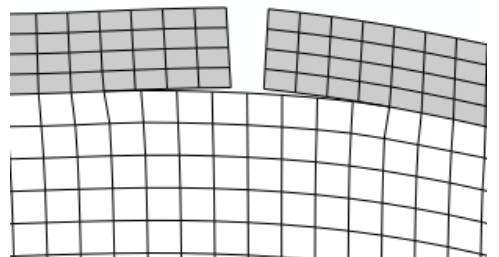

(c) On-Orbit (20x)

Figure 7. Deformed configurations for the Baseline-I case.

For on-orbit, the deformed configuration (at twenty times magnification) is shown in Figure 7(c). Note that at every location, due to the cold temperature, the two coating islands on either side of the craze crack surface contract away from each other, and the relative motion of the top and bottom defect surfaces is a sliding motion. The left and right tip $G_{\mathrm{T}} / G_{\mathrm{I}}$ values for the Baseline-I model subjected to the on-orbit loading condition are 1.6 and 1.5 , respectively. Therefore, this condition requires further examination. If $G_{\mathrm{T}}$ is Mode I-dominated, then the defect is unstable and likely to grow.

\section{Defect Location Parametric Study}

To study the effects of the interface defect location along the joggle, the interface defect is moved in 0.125-in. increments to the different craze crack locations shown in Figure 8 (i.e., from Location 0 (Baseline-I) to Location +1 , then Location +2 , etc.).

Lift-Off: Because the $G_{\mathrm{T}} / G_{\mathrm{I} c}$ values obtained for the Baseline-I model are essentially zero, the lift-off loading condition is considered to be benign. Hence, no further analyses are performed for interface defects subjected to the lift-off loading condition.

Entry: The interface defect locations shown in Figure 8 are analyzed for the entry loading condition. The results are shown in Figure 9. The deformed configurations for each defect location are shown at the top of the figure. The $G_{\mathrm{T}} / G_{\mathrm{Ic}}$ values for the left and right defect tips are plotted at the bottom of the figure as a function of the horizontal distance from Location 0. For the defects at locations within the joggle curve (vs. approaching the acreage), the $G_{\mathrm{T}} / G_{\mathrm{I} c}$ values for the right tips are much higher than the $G_{\mathrm{T}} / G_{\mathrm{I} c}$ values at the left tips, with the highest value at the right tip of the defect at Location $+2, G_{\mathrm{T}} / G_{\mathrm{Ic}}=1.9$. The $G_{\mathrm{T}} / G_{\mathrm{I} c}$ values for the right tips of the defects at Locations $+1,+2,+3$, and +4 are greater than unity, and hence, these defects for this condition require further examination. If $G_{\mathrm{T}}$ is Mode I-dominated, then the defect is unstable and likely to grow. The $G_{\mathrm{T}} / G_{\mathrm{I} c}$ values for the remaining defect tips are less than unity, and hence, these defects are stable and unlikely to grow.

On-orbit: The interface defect locations shown in Figure 8 are analyzed for the on-orbit loading condition. The results are shown in Figure 10. The deformed configurations for each defect location are shown at the top of the figure. The $G_{\mathrm{T}} / G_{\mathrm{I} c}$ values for the left and right defect tips are plotted at the bottom of the figure as a function of the horizontal distance from Location 0 . The $G_{\mathrm{T}} / G_{\mathrm{I} c}$ values for both the left and right defect tips at every location are nearly constant, with the highest value at the left tip of the defect at Location $-1, G_{\mathrm{T}} / G_{\mathrm{I} c}=1.7$. The $G_{\mathrm{T}} / G_{\mathrm{I} c}$ values for all of the defect tips are greater than unity. Therefore, this condition requires further examination. If $G_{\mathrm{T}}$ is Mode I-dominated, then the defect is unstable and likely to grow. See Reference 2 for detailed analysis and discussion of this case.

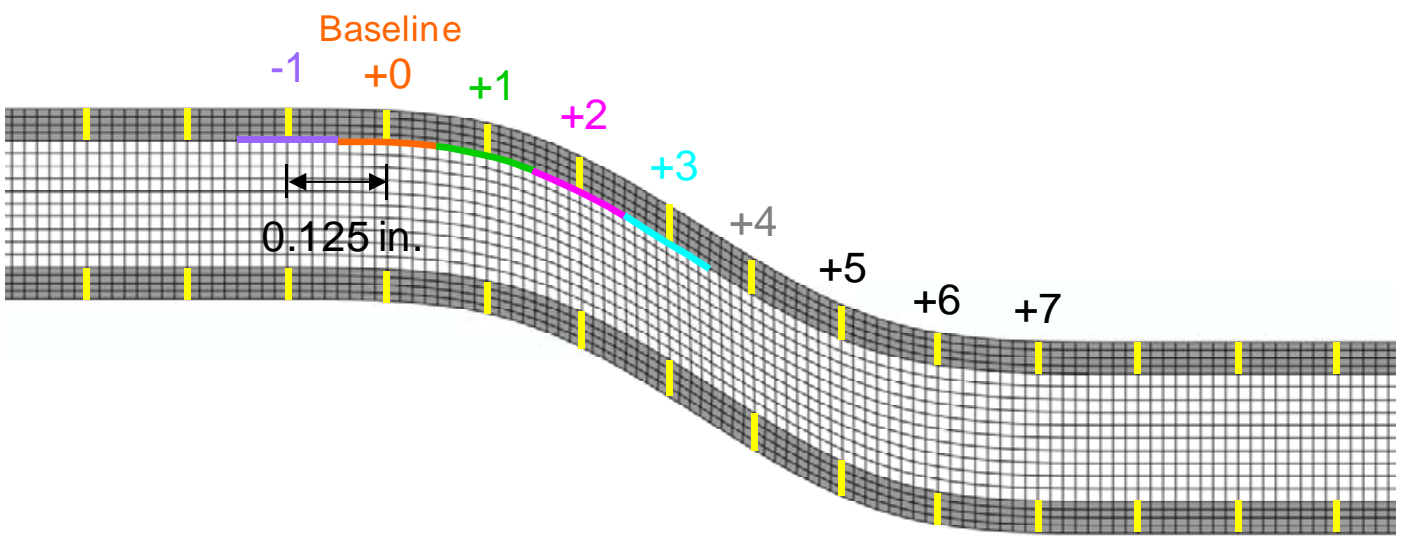

Figure 8. Single 0.125-in. interface defects at varying craze crack locations. 


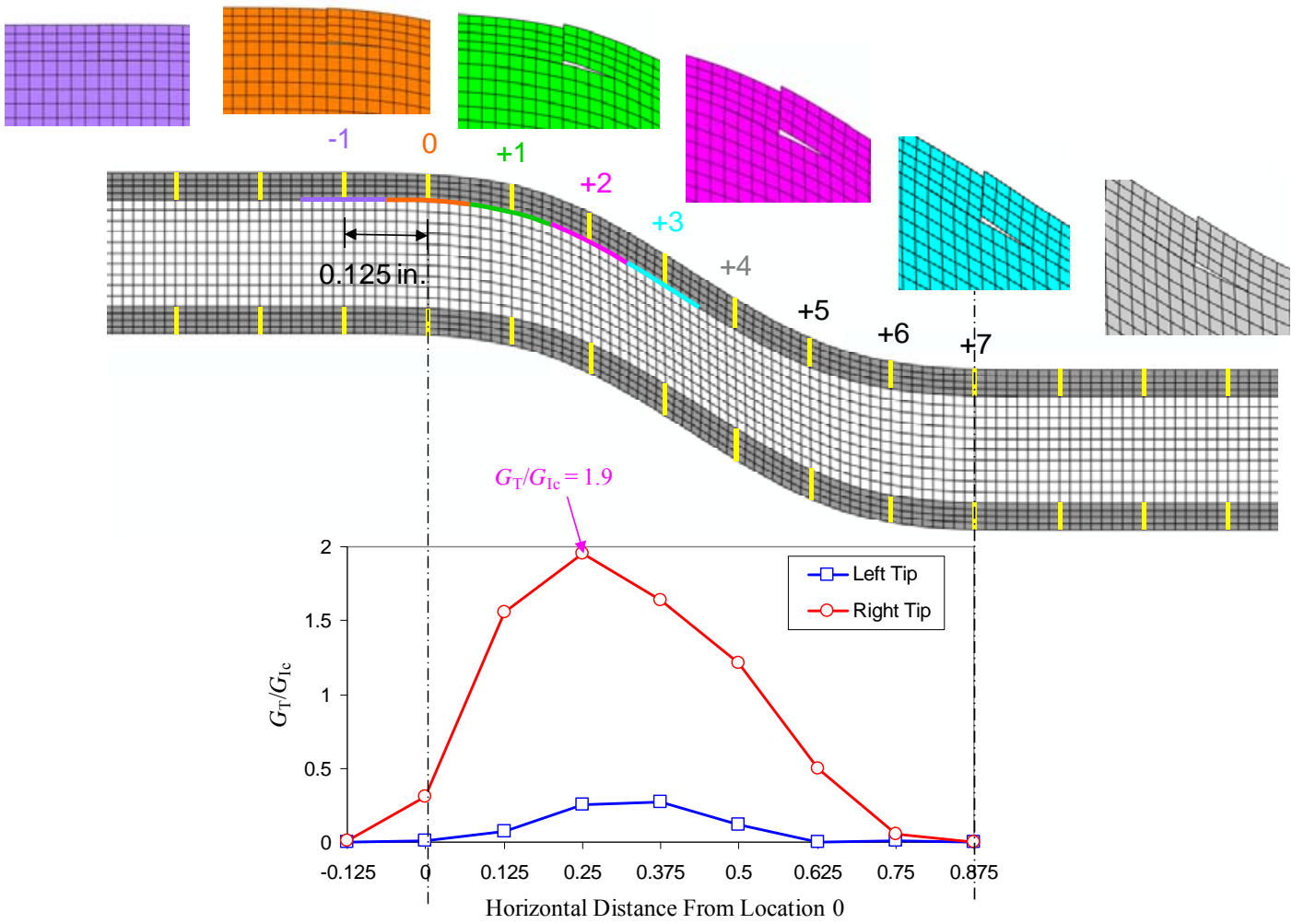

Figure 9. Entry deformed configurations and $G_{\mathrm{T}} / G_{\mathrm{Ic}}$ values for single 0.125 -in. interface defects at varying craze crack locations (deformations scaled by 10x).

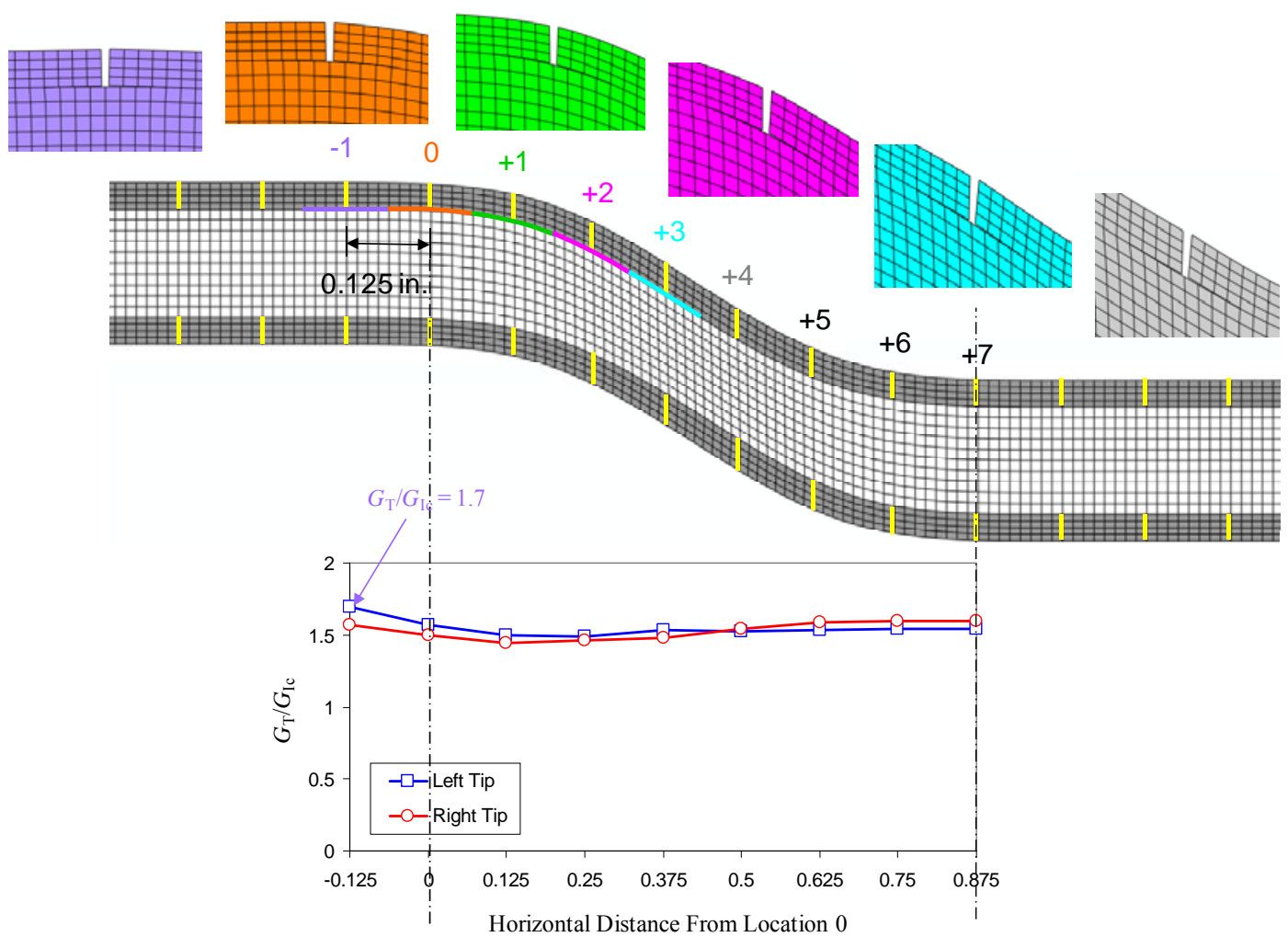

Figure 10. On-orbit deformed configurations and $G_{\mathrm{T}} / G_{\mathrm{Ic}}$ values for single 0.125 -in. interface defects at varying craze crack locations (deformations scaled by 10x).

American Institute of Aeronautics and Astronautics 


\section{B. Substrate Defects}

\section{Baseline-S Configuration}

The baseline configuration for substrate defects is denoted as Baseline-S for convenience in presentation. The Baseline-S model is shown in Figure 11. This model has a 0.125-in. substrate defect centrally located under the craze crack at Location 0, as shown in Figure 11(a). Because this defect is located within the substrate, the craze crack at this location remains connected at the coating-substrate interface, as shown in Figure 11(b).

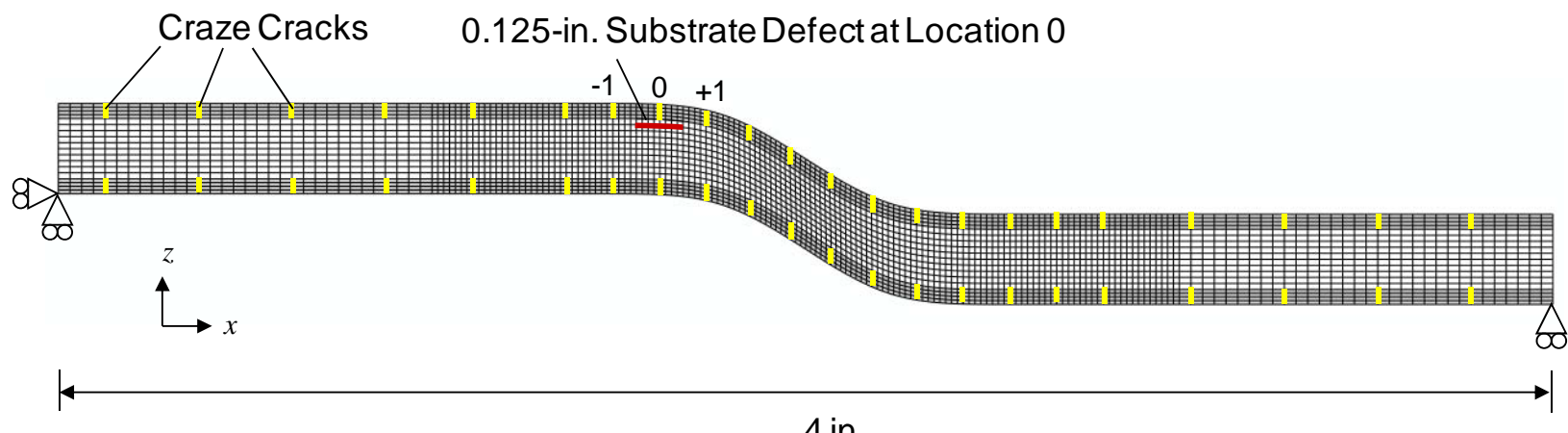

4 in.

(a) Finite element model

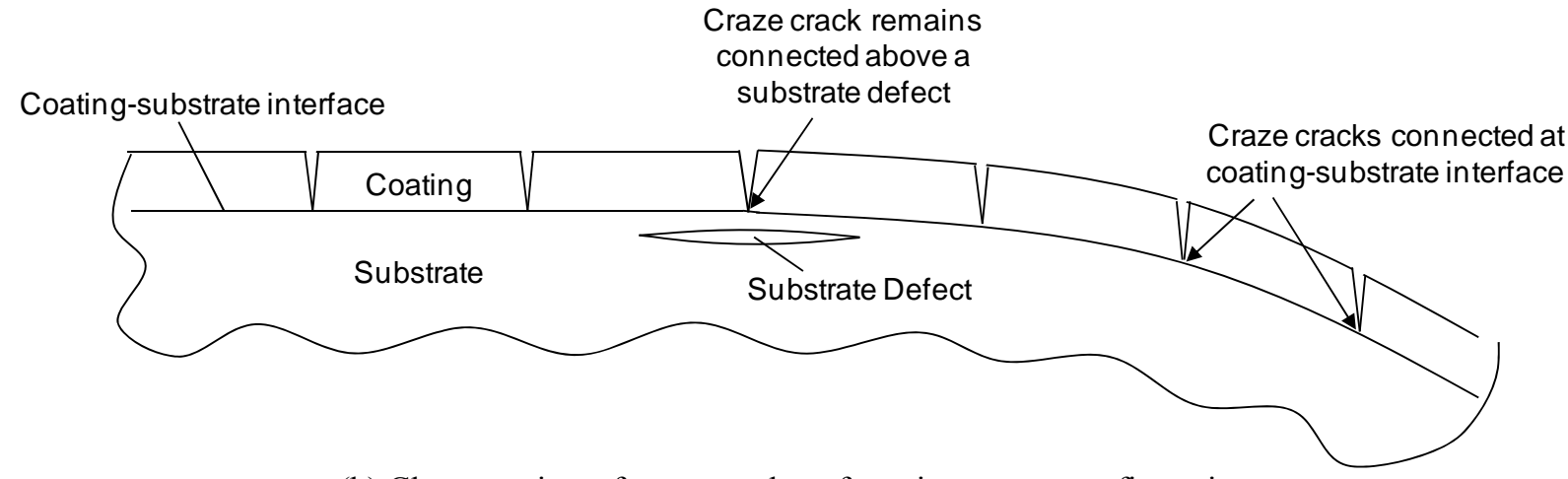

(b) Close-up view of craze crack surfaces in an open configuration

Figure 11. Baseline-S model.

For lift-off and ascent, the deformed configuration (at 500 times magnification) is shown in Figure 12(a). The pressure load pulls the coating material and the thin layer of substrate material between the defect and the interface upward (in the positive $z$ direction), opening the defect. Note that the additional stiffness provided by the thin layer of substrate material causes the substrate defect to open less than the interface defect, resulting in lower $G_{\mathrm{T}} / G_{\mathrm{Ic}}$ values than the $G_{\mathrm{T}} / G_{\mathrm{Ic}}$ values for the corresponding interface defect (compare to Baseline-I). Because the pressure load is applied normal to the coating surface, a small amount of bending is also observed. The left and right tip $G_{\mathrm{T}} / G_{\mathrm{Ic}}$ values for the Baseline-S model subjected to the lift-off loading condition are essentially zero (less than 0.001 ), indicating that the defect is stable and unlikely to grow.

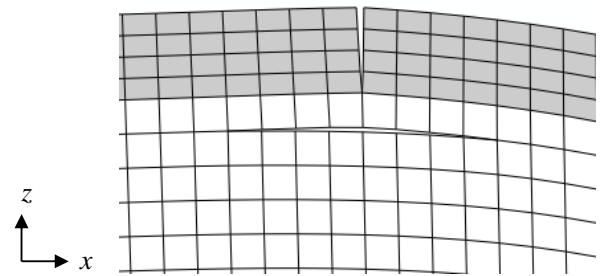

(a) Lift-Off (500x)

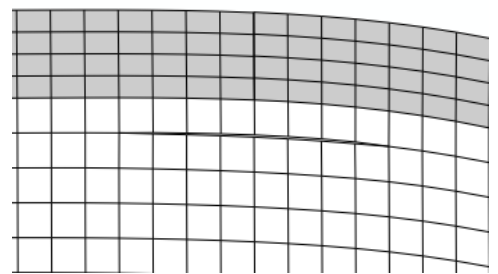

(b) Entry (20x)

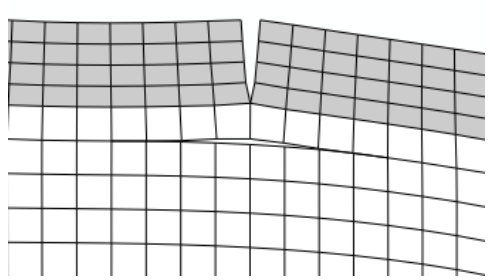

(c) On-Orbit (20x)

Figure 12. Deformed configurations for the Baseline-S case.

American Institute of Aeronautics and Astronautics 
For entry, the deformed configuration (at twenty times magnification) is shown in Figure 12(b). Due to the peak-heating temperature, as the coating material expands, the craze crack above the defect closes. In addition, some of the load is able to pass through the craze crack tip and the thin layer of substrate material into the left tip, resulting in left tip and right tip $G_{\mathrm{T}} / G_{\mathrm{I}}$ values that are much closer to the same value and much lower than the corresponding interface-defect-right-tip $G_{\mathrm{T}} / G_{\mathrm{Ic}}$ (compare to Baseline-I). The left and right tip $G_{\mathrm{T}} / G_{\mathrm{Ic}}$ values for the Baseline-S model subjected to the entry loading condition are 0.03 and 0.07 , respectively. Again, these near-zero values indicate that the defect is stable and unlikely to grow.

For on-orbit, the deformed configuration (at twenty times magnification) is shown in Figure 12(c). Note that the thin layer of substrate material prevents the craze crack above the defect from completely separating, reduces the sliding motion of the defect surfaces, and hence results in $G_{\mathrm{T}} / G_{\mathrm{IC}}$ values that are much smaller than the $G_{\mathrm{T}} / G_{\mathrm{Ic}}$ values for the corresponding interface defect (compare to Baseline-I). The left and right tip $G_{\mathrm{T}} / G_{\mathrm{Ic}}$ values for the Baseline-S model subjected to the on-orbit loading condition are 0.007 and 0.02 , respectively. Again, these nearzero values indicate that the defect is stable and unlikely to grow.

\section{Defect Length Parametric Study}

To study the effects of defect length, the long substrate defects shown in Figure 13 are considered. The 0.125 -in. substrate defect at Location 0 (Baseline-S) is shown in Figure 13(a). The long defect lengths considered are shown in Figures 13(b-d). For each of these defects, the left tip is fixed at Location $-1 / 2$, while the right tip changes position along the joggle. For the 0.1875-in. (Figure 13(b)), 0.25-in. (Figure 13(c)), and 0.375-in. (Figure 13(d)) defects, the right tips are at Locations $+1,+1 \frac{1}{2}$, and $+2 \frac{1}{2}$, respectively.

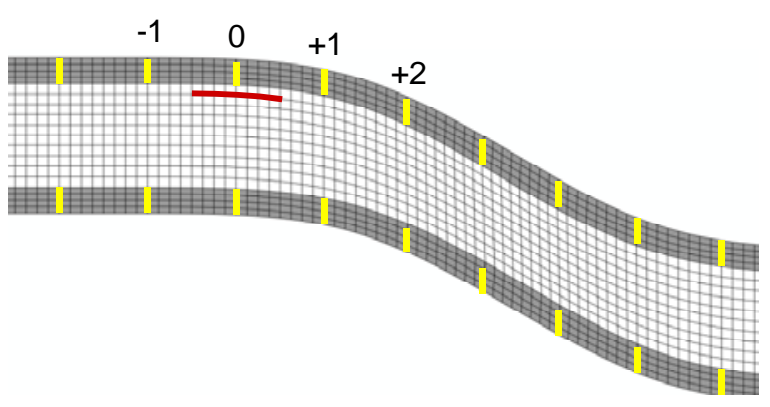

(a) 0.125 -in. substrate defect

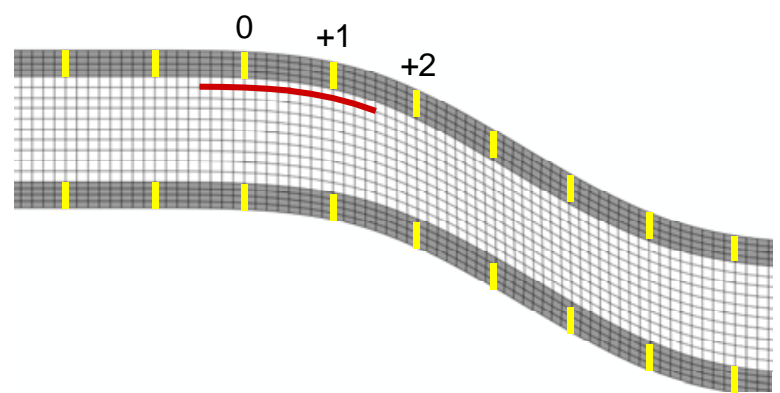

(c) 0.25 -in. substrate defect

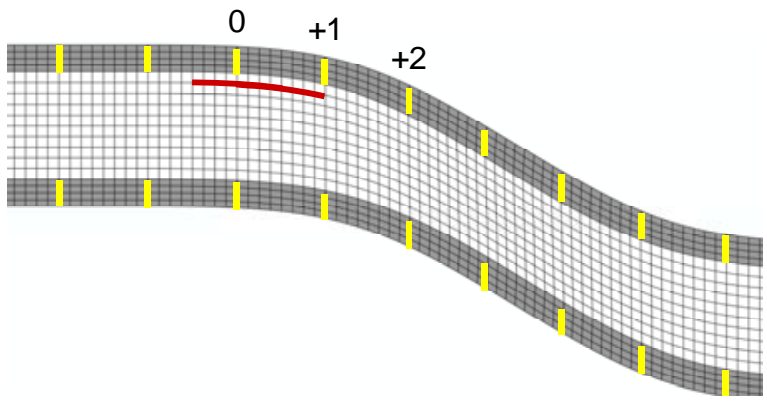

(b) 0.1875 -in. substrate defect

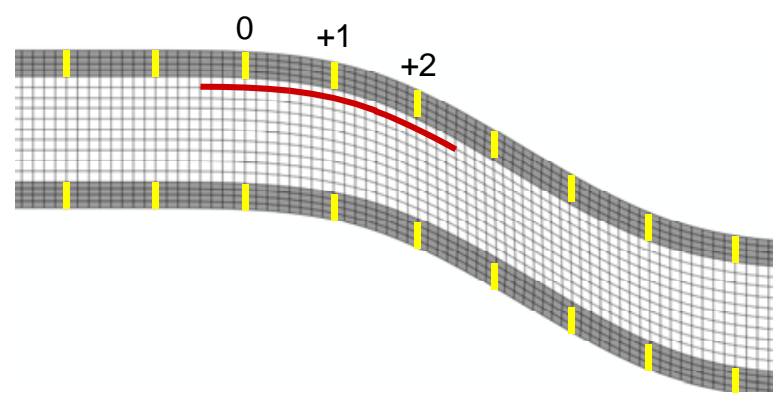

(d) 0.375 -in. substrate defect

Figure 13. Long substrate defects with the left tip fixed midway between Locations -1 and 0.

Lift-Off: Because the $G_{\mathrm{T}} / G_{\mathrm{Ic}}$ values obtained for the Baseline-S model are essentially zero, the lift-off loading condition is considered to be benign. Hence, no further analyses are performed for substrate defects subjected to the lift-off loading condition.

Entry: The long substrate defects shown in Figure 13 are analyzed for the entry loading condition. The $G_{\mathrm{T}} / G_{\mathrm{IC}}$ values for each of the long substrate defects are presented in Figure 14. As the defect length increases, the $G_{\mathrm{T}} / G_{\mathrm{Ic}}$ values for both the left and right tips also increase, with the $G_{\mathrm{T}} / G_{\mathrm{Ic}}$ values for the 0.375 -in. defect approaching a value of unity. Therefore, this condition requires further examination. If $G_{\mathrm{T}}$ is Mode I-dominated, then the defect is unstable and likely to grow.

On-orbit: The long substrate defects shown in Figure 13 are analyzed for the on-orbit loading condition. The $G_{\mathrm{T}} / G_{\mathrm{Ic}}$ values for each of the long substrate defects are presented in Figure 15. The right tip of the 0.1875-in. defect is located directly under a craze crack. This configuration causes the craze crack to interact with the defect tip, 
resulting in larger opening and a $G_{\mathrm{T}} / G_{\mathrm{Ic}}(0.0627)$ that is more than three times larger than the $G_{\mathrm{T}} / G_{\mathrm{Ic}}$ values for the other right tips $(\sim 0.02)$. However, the $G_{\mathrm{T}} / G_{\mathrm{Ic}}$ values for all defect tips are much less than unity, and hence, the defects are stable and unlikely to grow.

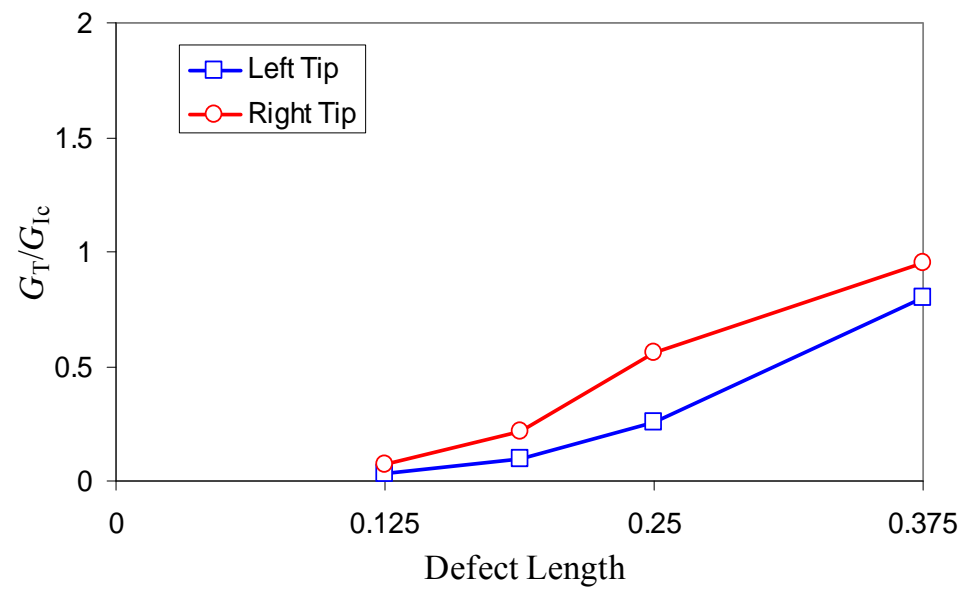

Figure 14. Entry $G_{\mathrm{T}} / G_{\mathrm{Ic}}$ values for long substrate defects with the left tip fixed.

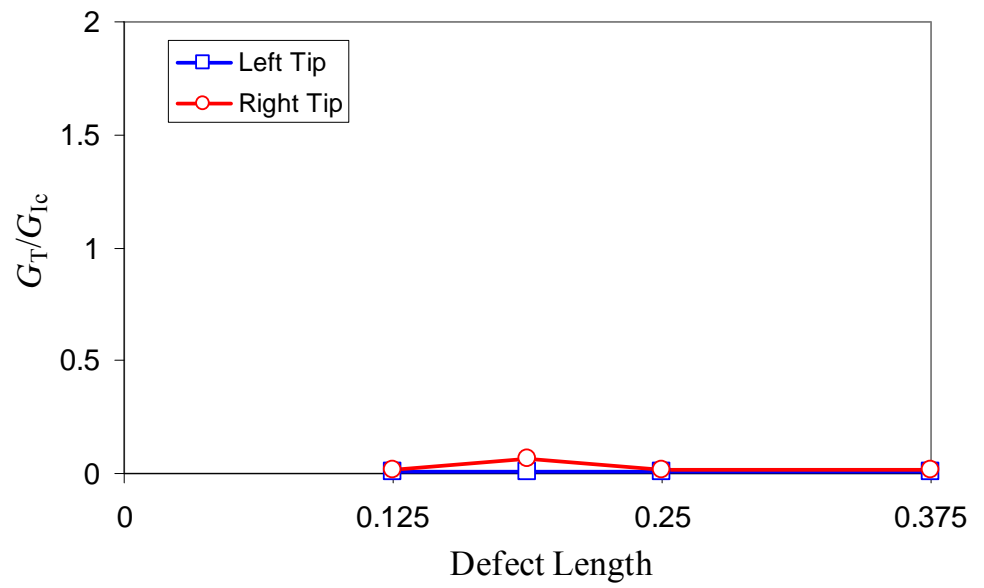

Figure 15. On-orbit $G_{\mathrm{T}} / G_{\mathrm{Ic}}$ for long substrate defects with the left tip fixed.

\section{Concluding remarks}

In this paper, fracture mechanics analyses of subsurface defects within the joggle regions of the Space Shuttle wing-leading-edge RCC panels are performed. A 2D plane strain idealized joggle model is developed to study the fracture behavior of the panels under three phases of flight - lift-off and ascent, on-orbit, and entry.

For the lift-off and ascent phase of flight, the panels experience mechanical loads and negligible thermal loading, and an estimated bounding pressure load is used for the analyses. For the entry and on-orbit phases of flight, thermo-mechanical analyses are performed using the extreme hot and cold temperatures experienced by the panels.

The analyses assume that subsurface defects are present either at the coating-substrate interface or in the substrate. The objective of the fracture mechanics analyses is to evaluate the defect driving forces, which are characterized by the strain energy release rates, and determine if defects can become unstable.

Based on the analyses in this paper, the following conclusions are drawn:

- The $G_{\mathrm{T}} / G_{\mathrm{Ic}}$ values for interface defects are larger than values for substrate defects.

- Of the three loading cases studied, lift-off and ascent loading yields the lowest $G_{\mathrm{T}} / G_{\mathrm{Ic}}$ values by several orders of magnitude. The $G_{\mathrm{T}} / G_{\mathrm{Ic}}$ values are much less than unity (essentially zero); therefore, unstable defect growth is very unlikely, and the lift-off loading condition is considered to be benign. 
- For entry:

- For interface defects, the right tip of an interface defect within the joggle region yields the largest $G_{\mathrm{T}} / G_{\text {Ic }}$ compared to other defect locations.

o For substrate defects, the $G_{\mathrm{T}} / G_{\mathrm{Ic}}$ values for the left and right defect tips increase with increasing substrate defect length.

- For on-orbit:

o For interface defects, both the left and right defect tips for all defect locations have nearly constant $G_{\mathrm{T}} / G_{\mathrm{IC}}$ values.

o For substrate defects, all defect tips have very small $G_{\mathrm{T}} / G_{\mathrm{Ic}}$ values.

- For entry and on-orbit, depending on defect location and length, the $G_{\mathrm{T}} / G_{\mathrm{Ic}}$ values may be greater than unity; therefore, these conditions require further examination. If $G_{\mathrm{T}}$ is Mode I-dominated, then the defect is unstable and likely to grow.

\section{References}

${ }^{1}$ Raju, I. S., Phillips, D. R., Knight, N. F., and Song, K. (2009a): "Fracture Mechanics Analyses of Idealized Acreage and Joggle Regions of Space Shuttle Orbiter Wing-Leading-Edge RCC Panels - Lift-Off and Ascent Loading," NASA/TM-2009-215923.

${ }^{2}$ Raju, I. S., Phillips, D. R., Knight, N. F., and Song, K. (2009b): "Fracture Mechanics Analyses of Idealized Acreage and Joggle Regions of Space Shuttle Orbiter Wing-Leading-Edge RCC Panels - On-Orbit and Entry Thermal Loading," NASA/TM-2009-215924.

${ }^{3}$ Knight, N. F., Jr., Song, K., and Raju, I. S. (2009a): "Global Thermo-Mechanical Stress Analysis of Space Shuttle Orbiter Wing-Leading-Edge Panels," NASA/TM-2009-215925.

${ }^{4}$ Knight, N. F., Jr., Song, K., and Raju, I. S. (2009b): "Factors Influencing Thermo-Mechanical Elastic Response of Slip-Side Joggle Regions of the Space Shuttle Orbiter Wing-Leading-Edge Panels," NASA/TM-2009-215926.

${ }^{5}$ Anon. (2007): ABAQUS Analysis User's Manual: Volumes I - VI, Version 6.7, Dassault Systèmes Simulia Corp., Providence, RI.

${ }^{6}$ Rybicki, E. F. and Kanninen, M. F. (1977): "A Finite Element Calculation of Stress Intensity Factors by a Modified Crack Closure Integral,” Engineering Fracture Mechanics, Vol. 9, pp. 931-938.

${ }^{7}$ Raju, I. S. (1987): "Calculation of Strain-Energy Release Rates with Higher Order and Singular Finite Elements," Engineering Fracture Mechanics, Vol. 28, pp. 251-274.

${ }^{8}$ Krueger, R. (2004): "The Virtual Crack Closure Technique: History, Approach and Applications," Applied Mechanics Reviews, 57, (2), pp. 109-143.

${ }^{9}$ Vaughn, W. L., Walker, S., and Koenig, J. (2008): Stress Free Temperature Determination for Reinforced Carbon-Carbon (RCC), NASA LaRC technical white paper, Version 4.0, October 23, 2008.

${ }^{10}$ Raju, I. S., Phillips, D. R., and Knight, N. F., Jr. (2009c): "Stress-Free Temperature Prediction for Reinforced Carbon-Carbon," NASA/TM-2009-215919. 